\title{
A perfect Fresnel acoustic reflector implemented by a Fano-resonant metascreen
}

M. Amin, O. Siddiqui, M. Farhat, and A. Khelif

Citation: Journal of Applied Physics 123, 144502 (2018); doi: 10.1063/1.5012012

View online: https://doi.org/10.1063/1.5012012

View Table of Contents: http://aip.scitation.org/toc/jap/123/14

Published by the American Institute of Physics

\section{Articles you may be interested in}

Perspective: Acoustic metamaterials in transition

Journal of Applied Physics 123, 090901 (2018); 10.1063/1.5007682

Inherent losses induced absorptive acoustic rainbow trapping with a gradient metasurface

Journal of Applied Physics 123, 091702 (2018); 10.1063/1.4997631

Broadband acoustic focusing by cavity structures with phase manipulations

Journal of Applied Physics 122, 244501 (2017); 10.1063/1.4998223

Acoustic metamaterial panel for both fluid passage and broadband soundproofing in the audible frequency range Applied Physics Letters 112, 041903 (2018); 10.1063/1.5004605

Broadband manipulation of refracted wavefronts by gradient acoustic metasurface with V-shape structure Applied Physics Letters 111, 263501 (2017); 10.1063/1.5005950

Systematic design of broadband path-coiling acoustic metamaterials

Journal of Applied Physics 123, 025101 (2018); 10.1063/1.5009488

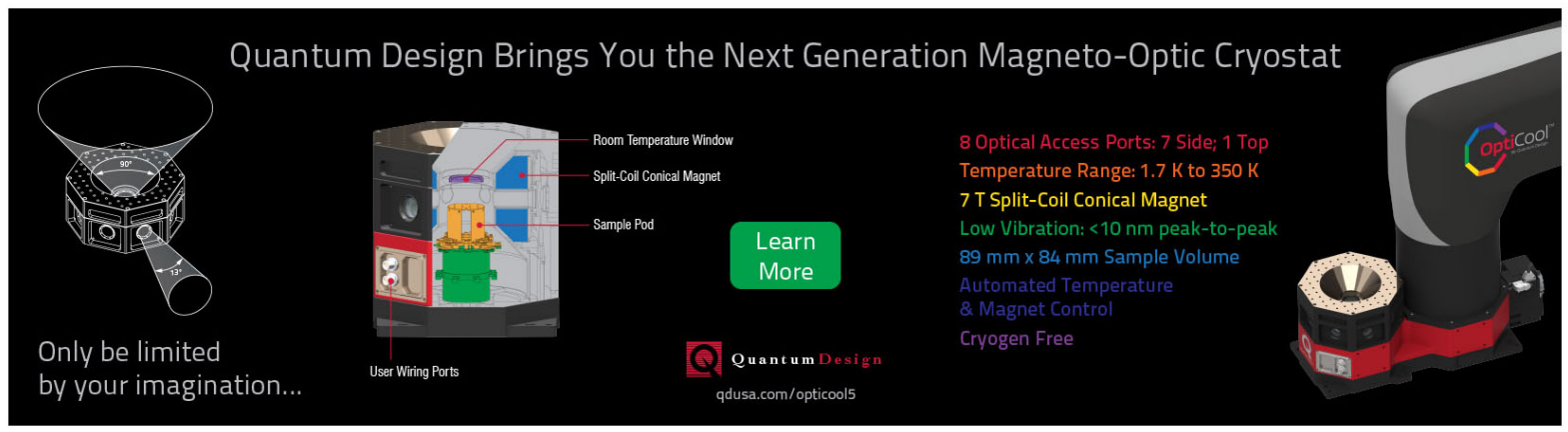




\title{
A perfect Fresnel acoustic reflector implemented by a Fano-resonant metascreen
}

\author{
M. Amin, ${ }^{1}$ O. Siddiqui, ${ }^{1}$ M. Farhat, ${ }^{2}$ and A. Khelif ${ }^{3}$ \\ ${ }^{1}$ College of Engineering, Taibah University, Madinah, Saudi Arabia \\ ${ }^{2}$ Division of Computer, Electrical, and Mathematical Sciences and Engineering, King Abdullah University \\ of Science and Technology (KAUST), Thuwal 23955-6900, Saudi Arabia \\ ${ }^{3}$ Institut FEMTO-ST, CNRS, Université de Bourgogne Franche-Comté, 15B Avenue des Montboucons, \\ F-25030 Besançon, France
}

(Received 4 November 2017; accepted 26 March 2018; published online 10 April 2018)

\begin{abstract}
We propose a perfectly reflecting acoustic metasurface which is designed by replacing the curved segments of the traditional Fresnel reflector by flat Fano-resonant sub-wavelength unit cells. To preserve the original Fresnel focusing mechanism, the unit cell phase follows a specific phase profile which is obtained by applying the generalized Snell's law and Fermat's principle. The reflected curved phase fronts are thus created at the air-metasurface boundary by tailoring the metasurface dispersion as dictated by Huygens' principle. Since the unit cells are implemented by sub-wavelength double slit-shaped cavity resonators, the impinging sound waves are perfectly reflected producing acoustic focusing with negligible absorption. We use plane-wave solution and full-wave simulations to demonstrate the focusing effects. The simulation results closely follow the analytical predictions. Published by AIP Publishing. https://doi.org/10.1063/1.5012012
\end{abstract}

\section{INTRODUCTION}

In the last few decades, there has been a growing interest in wave propagation in periodically structured electromagnetic media. ${ }^{1}$ The active investigation of the so-called photonic crystals has paved the way towards the theoretical prediction and subsequent experimental validation of photonic bandgap structures. ${ }^{2-7}$ In the same vein, studies on acoustic and classical mechanical waves in periodic media were put forward, and the existence of phononic bandgaps has been revealed both theoretically and experimentally. ${ }^{8-19}$ Several novel and unexpected phenomena have been put forward, such as acoustic invisibility cloaks, which could be used for seismic protection or undetectability of submarines, by using anisotropic effective physical parameters (density and bulk modulus). ${ }^{20-25}$ The other equally important application of acoustic metamaterials and phononic crystals is certainly the possibility of perfect lensing with super-resolution (that overcomes the diffraction limit). In particular, a slab of double negative acoustic metamaterial ${ }^{26-29}$ can image both the far-field propagating modes and the near-field modes of a point source, and consequently act as a perfect lens whose resolution is not limited not only by the wavelength of the radiation but also by the dissipation and imperfections in the used materials. ${ }^{16,30}$ Quite remarkably, the equations governing the dynamics of acoustic waves were shown to be equivalent to the Maxwell equations in the two-dimensional (2D) case allowing us to draw analogies between the two types of waves. $^{31}$ The Electromagnetically induced transparency $(\text { EIT })^{32-34}$ and the associated Fano resonances ${ }^{35,36}$ are wellestablished areas of optics research, recently investigated in the context of acoustics. ${ }^{37-39}$

The optical parabolic reflectors have historically been used in telescopes to focus the light rays coming from distant objects. In electromagnetics, parabolic reflectors have been widely implemented as high gain aperture antennas. ${ }^{40}$ Before the advent of modern communication systems, the acoustic mirrors of parabolic shape have extensively been used for sound amplification and concentration. Surprisingly, parabolic sound reflectors have been discovered at several historical buildings. ${ }^{41}$ More recently, with the introduction of the Huygens' metasurfaces, ${ }^{42}$ a renewed interest has been generated in dispersion-based electromagnetic and acoustic lenses and reflectors. ${ }^{43,44}$ The concept of the metasurface is rooted in the generalized Snell's law ${ }^{45}$ and the Huygens' principle, according to which some of the wavelets reaching the two-dimensional surface gain additional momentum than others so that the outgoing wavefronts can take desired shapes and directions. In other words, the unit cells (or metaatoms) are synthesized according to a specific dispersion profile to achieve various wave scattering manipulations. Huygens' metasurfaces have been exploited in various beam forming, beam steering, and focusing applications. ${ }^{46-48}$

The concept of this paper is inspired by the Fresnel lenses and reflectors which were conceived in order to compress the lens surface power onto a planar surface. ${ }^{49}$ The idea as illustrated in Figs. 1(a) and 1(b) is to preserve the optical power of the reflector by only removing the extra material while maintaining the surface curvatures. Hence, in both of the reflectors, i.e., the parabolic and its planar implementation, the focusing is obtained by physically fabricating the curved reflecting surfaces so that the Fermat's principle can be invoked in its original form. Here we propose to replace each Fresnel's curved segment with a smoothsurfaced resonant unit cell (meta-atom) that introduces an abrupt localized phase change such that the original direction of the reflected ray is preserved. These unit cells which are arranged in a two-dimensional geometry form a metasurface, as depicted in Fig. 1(c). Contrary to the parabolic shape 


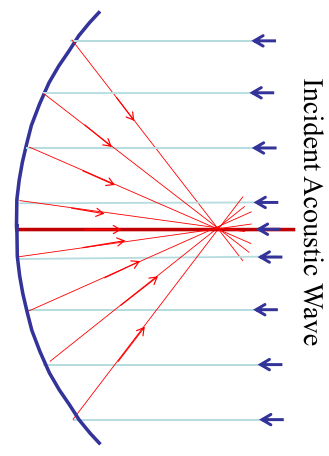

(a) Parabolic Reflector

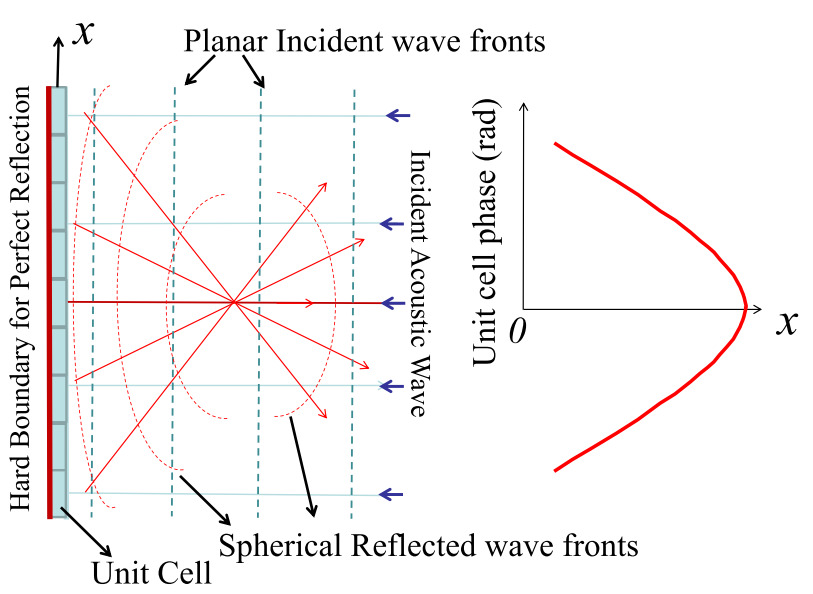

(c) Dispersive Fresnel Reflector

(d) Phase Profile

FIG. 1. Conceptual flow of the Fresnel metasurface idea. (a) The traditional parabolic reflector and (b) its implementation as conceived by Fresnel. (c) The acoustic metasurface designed to achieve a (d) parabolic phase profile. The wave fronts converge at the focal point on account of the dispersive phase.

reflector, the physical paths of all the rays arriving to the focal point of the metasurface have unequal lengths. The focusing is realized by tailoring the phase profile [Fig. 1(d)] so that spherical phase fronts are created at the interface of air and the metasurface.

The driving idea of the proposed metasurface is the tunable control of the phase of the reflection spectra from the metascreen by varying the geometry properties of its unit cells (meta-atoms). The most efficient Fresnel metasurface would have near unity reflection, zero absorption, and the unit cell dimensions that are not too different from the adjacent cell so that a flat geometrical profile can be maintained. Therefore, we propose air-filled slit-shaped quarter wavelength resonator cavities as unit cells with rigid acoustic material that invokes hard boundary conditions for the pressure waves to achieve near unity reflection. Such unit cells produce negligible absorption and are intrinsically impedance-matched to free space because of their continuous (without gaps) geometry. On the other hand, the other contemporary approaches of phase control such as the split ring resonators ${ }^{50}$ or the three dimensional space coils and the labyrinthine geometries ${ }^{51-53}$ employ noncontinuous complex structures leading to significant absorption losses. The gradient index acoustic lens ${ }^{54}$ suffers from impedance-mismatch and hence the focused pressure fields do not form a perfect image. Another important attribute of the proposed slitshaped geometry is the existence of strong resonant-coupling between the dual slits that results in Fano resonant line shape with an inherent sharp phase slope. Hence the phase characteristics can be tailored with slight modification in the dimensions of adjacent cells leading to extremely compact flat geometry. We anticipate that the proposed acoustic metasurface on account of its flatness can be effectively embedded in planar structures and walls.

\section{THE FRESNEL METASURFACE-RAY AND FOURIER ACOUSTICS}

To understand the focusing mechanism, the two dimensional ray diagram of the acoustic system with a metasurface of aperture $2 D$ is drawn in Fig. 2. All the acoustic rays impinging the air-metasurface boundary $A A^{\prime}$ as planar wavefronts would travel a distance $2 d^{\prime}$ to return to the same location after undergoing the reflection from the hard boundary. If the metasurface were not present, all the rays would have reached the interface $A A^{\prime}$ at the same time with same wave velocity forming planar reflected wavefronts, as required by the Fermat's principle. The metasurface is designed in such a way that each unit cell changes the impinging wavelet's momentum by slowing down some of the waves while accelerating the others. ${ }^{55}$ To achieve focusing, the time required for all the waves to reach the focal point $F$ should be equal. Knowing that the largest distance is traveled by the wave emanating from the farthest unit cell from the origin, the following condition can be written to equalize the required times:

$$
\frac{2 d^{\prime}}{v_{\min }}+\frac{O F}{c}=\frac{2 d^{\prime}}{v_{\max }}+\frac{A F}{c},
$$

where $O F$ is the distance from origin $O$ to focal point $F, c$ is the sound speed in air, $A F=\sqrt{F^{2}+D^{2}}$, and $v_{\min }$ and $v_{\max }$ are the minimum and maximum velocities exhibited by the waves traversing the unit cells that are, respectively, located at the center and at the edge of the metasurface. Equating $2 d^{\prime}=d$ and writing the wave velocities in terms of the phase constant $k$ and radian frequency $\omega$, Eq. (1) can be rewritten as

$$
\frac{k_{\max } d}{\omega}+\frac{k_{o} F}{\omega}=\frac{k_{\min } d}{\omega}+\frac{k_{o} \sqrt{D^{2}+F^{2}}}{\omega},
$$

where $k_{o}=2 \pi / \lambda$ is the free-space phase constant. Equation (2) can be simplified after omitting $\omega$ and substituting $k_{\text {max }} d$ $=\phi_{\max }$ and $k_{\min } d=\phi_{\min }$ which are the respective maximum and minimum phases on the air-metasurface interface

$$
\phi_{\max }+k_{o} F=\phi_{\min }+k_{o} \sqrt{D^{2}+F^{2}} .
$$

A similar equation can be written for a generalized acoustic path between any unit cell situated at $(x, 0)$ and the focus of the system

$$
\phi_{\max }+k_{o} F=\phi_{y}+k_{o} \sqrt{x^{2}+F^{2}} .
$$



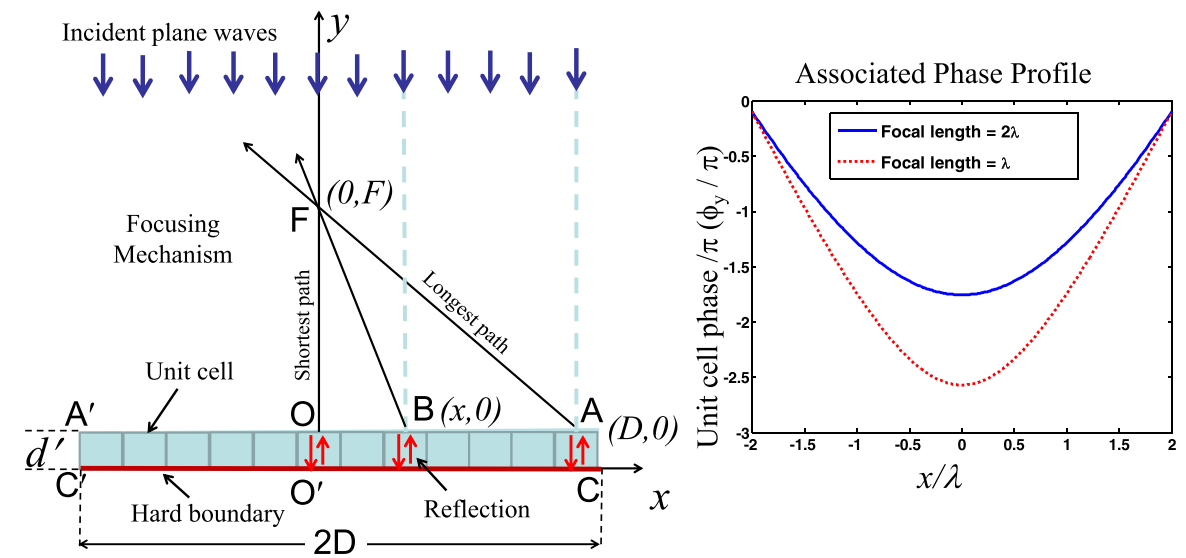

FIG. 2. Explanation of the focusing mechanism with the help of the ray diagram. The left side shows the parallel planar wavefronts as they are reflected by the flat Fresnel metascreen with the dispersion profile given on the right hand side. With the parabolic phase profile, the reflecting wavefronts converge on the focal point.
The acoustic dispersion equation can then be obtained by subtracting Eq. (3) from Eq. (4) and rearranging

$$
\phi_{y}=k_{o}\left(\sqrt{D^{2}+F^{2}}-\sqrt{x^{2}+F^{2}}\right)+\phi_{\text {min }} \text {. }
$$

Here $\phi_{y}$ is the phase incurred when the impinging wave traverses an arbitrary unit cell situated on the Fresnel metasurface. The design procedure starts with the specification of the aperture size $D$ and the desired focal length $F$. A suitable initial phase $\phi_{\text {min }}$, taking into consideration the design constraints, can be assigned to the last unit cell. The rest of the phases in the profile can be found from Eq. (5). Consider a representative design for a Fresnel metasurface of aperture $2 \lambda$ with a minimum phase of 0.3 radians. The dispersion profile for two focal lengths $2 \lambda$ and $\lambda$ is calculated from Eq. (5) and is depicted in Fig. 2. As anticipated from observing Eq. (5), the unit cells follow a parabolic dispersion which is an essential condition for focusing. For the closer focal points, the larger phase angle values per unit cell produces spherical wavefronts of smaller radii as dictated by the Huygens' principle and the Snell's law of reflection. In the practical design, a larger number of unit cells result in a smoother phase profile leading to higher resolution images. With fewer unit cells, there will be more abrupt changes in the phase profile which will then be piece-wise parabolic. Consequently, not all the details of the incoming sound will be captured resulting in a low resolution image.

To corroborate the metasurface focusing hypothesis presented above, a plane wave solution based on Huygens' principle can be formulated. Since perfect reflection from the hard boundary on the backside of the metasurface is assumed, a point source can be assumed on each unit cell which emits pressure fields in all directions. The total pressure field can then be evaluated in the image plane by the plane wave expansion method. ${ }^{56}$ Here since the plane wave sources are discrete, the Fourier integral becomes a summation of the contributions of all the plane wave sources of unit amplitudes. The pressure field on any point in the $x y$ plane can be written as

$$
P(x, y)=\sum_{n=-N / 2}^{+N / 2} e^{-j k_{o} \sqrt{\left(x-x_{n}\right)^{2}-y^{2}}} e^{-j \phi_{y}}
$$

where $N$ is the discrete number of plane wave sources and $x_{n}$ is the discrete location of individual source. To validate the design and its plane wave solution, a two dimensional Fresnel reflecting metasurface of aperture $4 \lambda$ and 30 number of unit cells is designed. The phase profile is obtained from Eq. (5) for the foci located at points $(0,2 \lambda)$ and $(0,4 \lambda)$. The corresponding pressure field amplitudes are then evaluated from Eq. (6). The phase profile and the normalized field distributions, depicted in Fig. 3, show the forming of the focus at the designed location for both of the phase profiles. The shift of the focus with the phase profile change shows that the metasurface can be mechanically tuned to obtain the sound amplification at a desired location. Not shown here is the case when a partial segment of the parabolic phase is exploited. In this case, the sound beam can be directed towards off-axis locations.

\section{THE UNIT CELL DESIGN WITH FANO RESONANCE CONCEPTS}

\section{A. The physical mechanism of the detuned Fano resonance}

The former class of Fano resonances was discovered almost half a century ago by Ugo Fano in the context of quantum mechanics, ${ }^{57}$ while investigating the inelastic scattering of electrons from helium. The Fano resonance line shape is obtained when destructive interference occurs between a resonant (discrete) process and another background process that consists of scattering within a continuum of states, leading to resonances with asymmetric line shapes. ${ }^{58}$ Owing to the fact that this is a general phenomenon, several applications in different fields have been put forward, e.g., switching, sensing, or in nonlinear optics. ${ }^{36,59-63}$ The dynamic control of amplitude and phase characteristics of optical waves was shown in graphene-based Fano resonances. ${ }^{59}$ In the framework of acoustics, several studies have been conducted to demonstrate acoustically induced transparency (AIT) and acoustic Fano resonance using optics-inspired techniques, such as the acoustic mode coupling with significantly different Q-factors. ${ }^{37-39}$ More recently, acoustic Fano resonances were studied in co-axial pipes with unequal lengths. ${ }^{39}$ The associated AIT effect was experimentally demonstrated in $\mathrm{kHz}$ spectrum with pipe sizes in the range of few millimeters. On account of their distinctively sharp phase profile, the Fano-resonators can be employed in geometrically flat compact metasurfaces where 

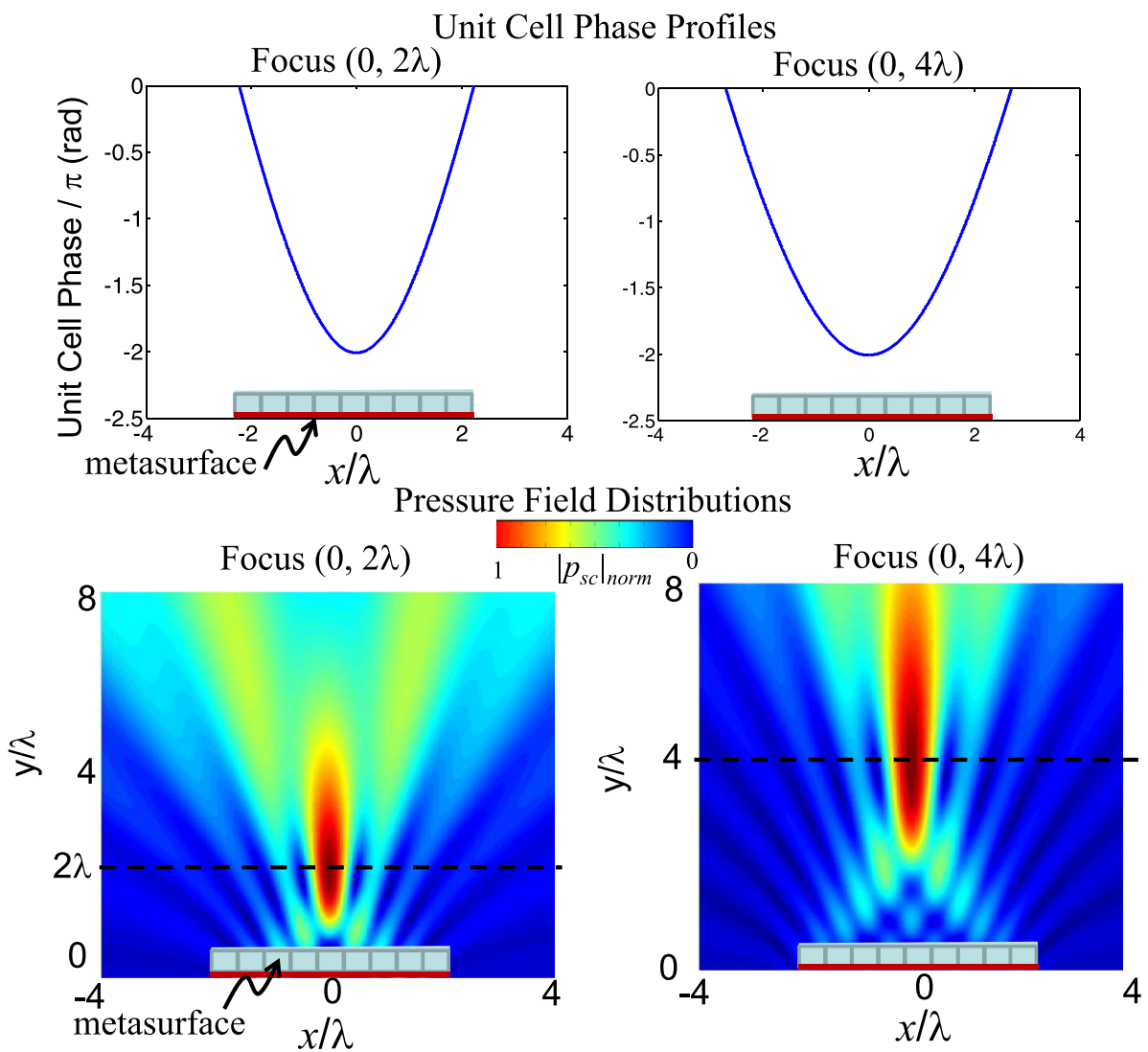

FIG. 3. The normalized pressure fields (second row) for the two different metasurface phase profiles given in the top row. The metasurface extends from $-2 \lambda$ to $2 \lambda$. The colorbar represents scattered pressure field distribution normalized between 0 and 1 . the adjacent cells are required to be slightly frequency-detuned from each with similar dimensions.

In order to tailor the phase profile of the proposed Fresnel metasurface, we first investigate its unit cell constituent, i.e., the slit-shaped resonator cavity $R_{1}$, shown in Fig. 4(a). The cavity, made of a rigid acoustic material, resonates on a frequency which is dependent on its shape and length. Here, as a representative case, we choose a slit-shaped cavity resonator of width and height as $21 \mathrm{~mm}$ and $186 \mathrm{~mm}$, respectively. The structure is implemented in the finite-element based COMSOL Multiphysics solver. The full-wave simulations are carried out for the case when sound waves traveling along the $y$-axis are normally incident on the isolated resonator $R_{1}$, which resides in the background 'air' medium. The computational domain is terminated with hard boundary conditions on the structure's surfaces along the y-axis and with the perfectly matched layers (PMLs) along the $\mathrm{x}$-axis to emulate the infinite simulation space. The scattered-field formulation is employed to simulate an incident wave source condition.
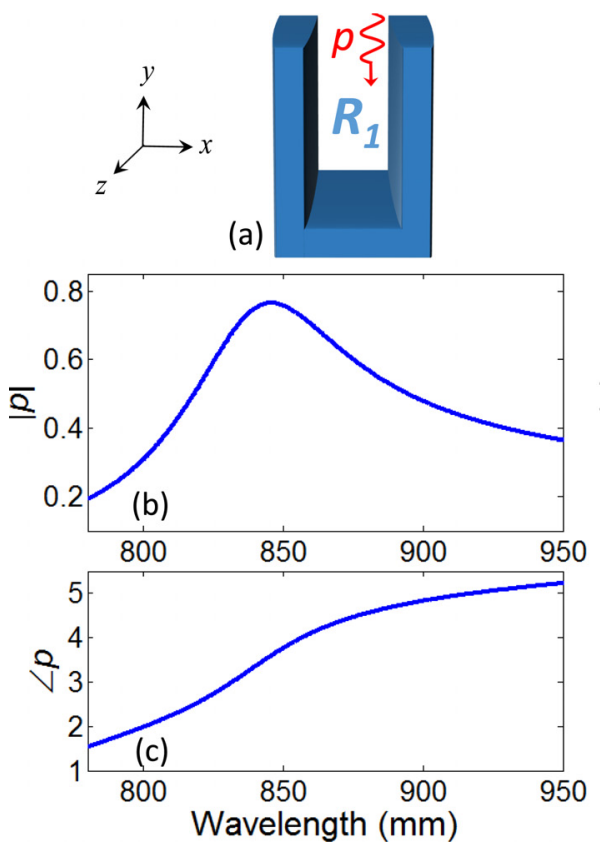

(d)
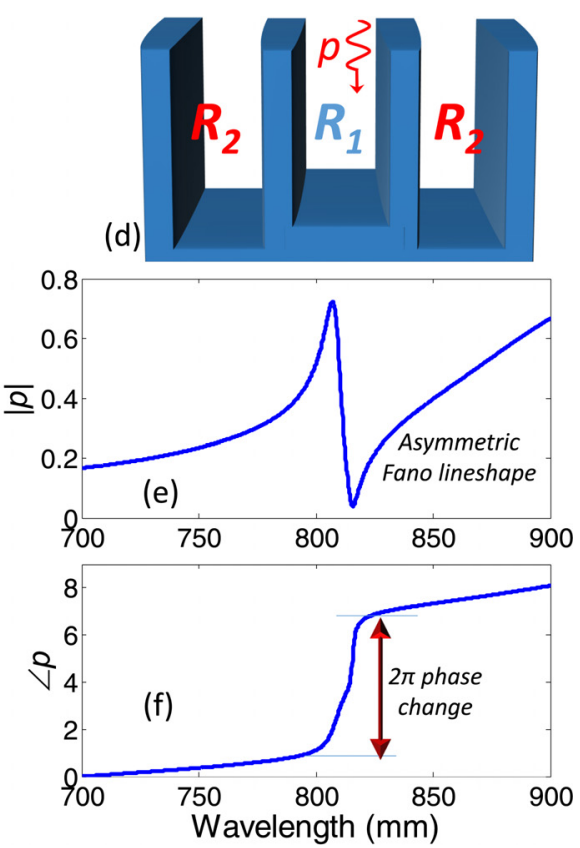

FIG. 4. (a) Schematic view of an isolated slit-shaped resonator $R_{1}$ and its (b) backscattering amplitude and (c) phase characteristics. The amplitude and phase characteristics are of typical symmetric Lorentzian line shape. (d) The Fano resonance is obtained by placing two detuned resonators $R_{1}$ and $R_{2}$ in proximity. The backscattering (e) amplitude and (f) phase characteristics show the Fano line shape. A sharp phase change of nearly $2 \pi$ is evident around resonant wavelength. 
Looking at the amplitude response of backscattered pressure field of single isolated resonator $R_{1}$ [Fig. 4(b)], it can be noted that the resonant spectral shape is nearly symmetric. Therefore, it can be related to conventional Lorentzian line shape with the resonance mode centered around a quarter wavelength, i.e., $\lambda_{0}=850 \mathrm{~mm}$. The phase spectrum of the resonator's backscattered field in Fig. 4(c) shows a smooth phase variation of less than $\pi$ radians across the Lorentzian line shape. This narrow phase variation range limits its capability in phase control applications.

To obtain a broader dynamic range, the symmetric Lorentzian resonance can be transformed to the Fano resonance by introducing a slightly detuned resonator $R_{2}$ (with an incremental length difference of $10 \mathrm{~mm}$ ) in the proximity of $R_{1}$, as depicted in Fig. 4(d). Note that in the simulation domain, two identical detuned resonators are added on either side to obtain a symmetric geometric scheme. The corresponding amplitude and phase spectra of the pressure fields are depicted in Figs. 4(e) and 4(f). Interestingly, due to the detuning effect, a sharp asymmetric Fano line shape around the quarter wavelength of $\lambda_{0}=810 \mathrm{~mm}$ is observed. The formation of the Fano resonance can be explained by first considering the fact that the slightly larger cavity produces a red-shifted resonance compared to that of the smaller one. In addition, the total backscattering field add in-phase (constructive interference) at the wavelength below $\lambda_{0}=810 \mathrm{~mm}$ and out-of-phase (destructive interference) at the wavelength $\lambda_{0}=810 \mathrm{~mm}$. The phase spectrum of backscattered field in Fig. 4(f) exhibits a sharp $2 \pi$ phase change across the Fano resonance. This specific amplitude-phase relation is in fact consistent with the well-known Kramers-Kronig relationship, which states that the sharp contrast in the phase of backscattered field is proportional to the difference between the extrema of the amplitude response. ${ }^{64}$ It should be emphasized here that the full $2 \pi$ phase variation of the scattered field provides the necessary phase flexibility to design the flat-surfaced sub-wavelength unit cells that constitute the Fresnel metasurface.

\section{B. Reflection properties of a periodic slit-shaped resonator array}

Next consider the unit cell that consists of the coupled Fano structures $R_{1}$ and $R_{2}$ [depicted in Fig. 4(d)] repeated periodically over a period of ' $a$ ' to form an infinite slitshaped resonator array, as depicted in Fig. 5(a). The geometric parameters $h_{1}, h_{2}, r_{1}$, and $r_{2}$ [Fig. 5(b)] that represent the heights and widths of the two cavities can be changed to tune the desired frequency response. To demonstrate the reflection properties of a representative case, the infinite structure with a relative height ratio $s=h_{2} / h_{1}=1$ and a width ratio $r_{1} / r_{2}=2 / 7$ is implemented in COMSOL. The reflectance simulations of the periodic structure illuminated by the pressure waves reveal a striking result. Since the rigid ground totally suppresses the transmitted fields, a complete reflection of the incident pressure fields $(|R(\omega)|=1)$ is observed in Fig. 5(c). More interestingly, as depicted in Fig. 5(d), the reflected field still maintains Fano's signature phase profile. This seemingly counter-intuitive effect of perfect amplitude response accompanied by an abrupt phase change can be explained by understanding the fact that both the resonant and non-resonant fields are equally reflected from the periodic slit array. Therefore, it is difficult to distinguish between the two types of fields in the amplitude spectrum. This important property of the resonator array is exploited in Sec. III C to tailor the phase response that is essential for the Fresnel metasurface.

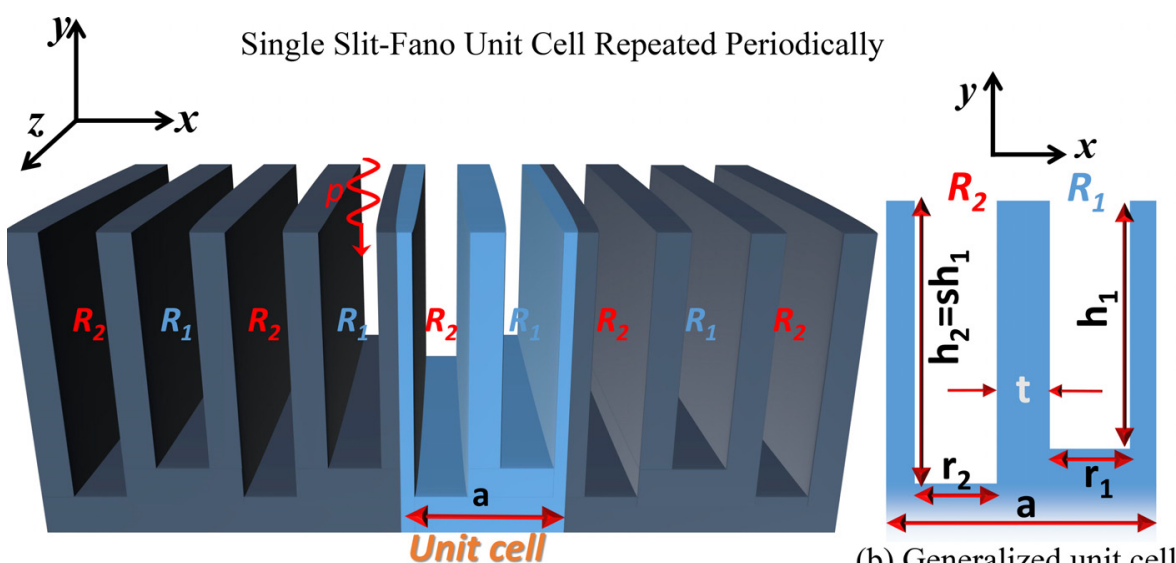

(a) Periodic structure

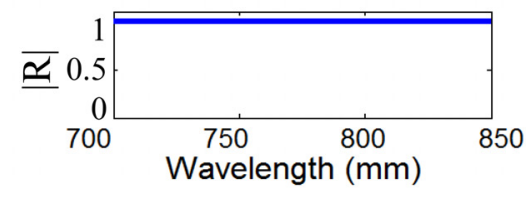

(c) Reflectance Magnitude

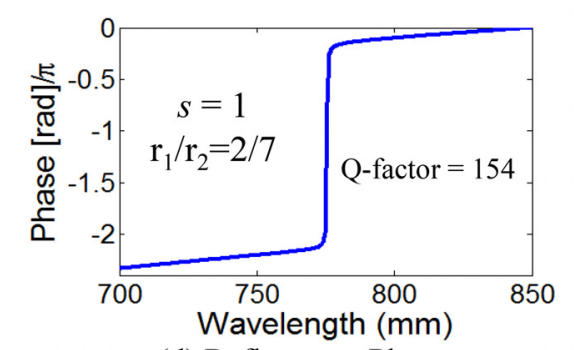

(d) Reflectance Phase
FIG. 5. (a) A schematic view of the periodic Fano resonator array (on top of a rigid ground). (b) The unit cell of the Fano resonator array which consists of two resonators $R_{1}$ and $R_{2}$. The period of the unit cell is $a=7 \mathrm{~cm}$. The geometrical parameters of the Fano Resonator surface are as follows: resonator height $h_{1}=8 / 3 a$, height $h_{2}=s h_{1}$ scaled relative to $h_{1}$, width of cavities $r_{1}, r_{2}$, and wall thickness $t=1 / 5 a$. (c) Reflectance amplitude when the array is illuminated normally by pressure fields showing a perfect reflection $|R|=1$. (d) Reflection phase for the representative case showing Fano phase profile. 


\section{Parametric study of the periodic resonator array}

Ideally, a smoothly varying phase with a somewhat lower Q-factor [compared to Fig. 5(d)] is preferred in order to provide larger bandwidth and cushion for fabrication precision in the metasurface design. At this point, a parametric study is imminent to optimize the phase response. We realize two different ways to detune the resonators to achieve Fano resonance, i.e., varying the relative widths of the two resonators contained in the array unit cell $\left(r_{1} / r_{2}\right)$ or their respective heights $\left(s=h_{2} / h_{1}\right)$.

First, consider the phase variation due to relative width scaling $\left(r_{1} / r_{2}\right)$ while assuming equal heights, i.e., $s=1$. If the widths are also assumed to be equal $\left(r_{1} / r_{2}=1\right)$, the two resonators become fully tuned and the destructive interference necessary for Fano effect cannot take place leading to no differential phase change in the reflection spectrum [Fig. 6(a)]. When the width of the resonator $r_{1}$ is narrowed relative to $r_{2}$, the $2 \pi$ phase variation is obtained in the reflection spectrum, as shown in Fig. 6(b). Decreasing the width $\left(r_{1} / r_{2}=0.64,0.38,0.2\right)$ also leads to a red-shift in the resonances, see Fig. 6(b).

Second, we characterize the effect of the relative height scaling $\left(s=h_{2} / h_{1}\right)$ on the phase response of the resonator array. The relative width $\left(r_{1} / r_{2}=2 / 7\right)$ of the resonators is chosen to facilitate maximum detuning effect and achieve a suitable Q-factor supported by the slit resonator array. Figure 6(c) plots the variation in the resulting reflection phase spectrum showing the red-shift as the relative height of resonator cavities is continuously scaled between $s=0.8$ and 1.2 .

Figure 6(c) summarizes the effect of changing the scaling factor $s$ on the phase profile of the Slit array for different incident wavelengths, while keeping the width scaling fixed at $r_{1} / r_{2}=2 / 7$. As observed, the resonator array can be tuned to an arbitrary phase shift by simply varying the scaling factor $s$. Consider the effect on the resonance bandwidth (Q-factor) by observing the two special cases of reflection phase response, i.e., $s=1$ and 1.2, given, respectively, in Figs. 5(d) and $6(\mathrm{~d})$. For $s=1$, the minor detuning due to the width scaling of $r_{1} / r_{2}=2 / 7$ leads to the narrow-band response resulting in a very high Q-factor around 154 [Fig. 5(d)]. On the other hand, a larger detuned height parameter $(s=1.2)$ results in a slowly varying phase response with a lower Q-factor of around 8, as shown in Fig. 6(d).

Note that the design of metasurface requires many of these slit-shaped resonators with a wide range of phases placed sequentially. Therefore, it is instructive from the design viewpoint to characterize the phase relationship for single wavelength. Figure 7(a) depicts the change in the reflectance phase extracted from Fig. 6(c), when the scaling parameter of the slitshaped array is varied at an incident wavelength of $790 \mathrm{~mm}$. Full range of phase angles $(0-2 \pi)$ is obtained by a differential geometric variation $(s=1-1.2)$, which is attributed to the inherent characteristics of the Fano resonance. Moreover, a large ratio between operating wavelength and the resonator period, i.e., $\lambda / a=790 \mathrm{~mm} / 70 \mathrm{~mm}=11.28$ allows fine discretization of the Fresnel phase profile function of Fig. 2. Both of these characteristics of the slit-shaped array lead to the design of compact flat-profiled metasurfaces.

Finally, the interference characteristics of the longitudinal resonances are used to understand the formation of Fano line shapes in the slit-shaped cavities. Consider in Fig. 7(b) the scattered field distribution across the proposed unit cell at several scaled heights $s$, which are marked in Fig. 7(a). For the scaling factor of $s=0.8$, high pressure field builds inside the narrow resonator cavity $R_{1}$ compared to $R_{2}$. The resonator cavity $R_{2}$ does not resonate around this scaling factor. Around scaling height $s=1$, a high pressure field builds inside the resonator cavity $R_{2}$ compared to $R_{1}$, while the pressure fields remain out-of-phase between two cavities. Interestingly, the scaling factor $s=1.2$ leads to a phase reversal of the pressure fields between the cavity and therefore prompts the destructive interference necessary for Fano resonance as seen in Fig. 4(e). As a result, a sharp phase change of $s=1-1.2$ in the reflection spectrum [Fig. 7(a)] of nearly $2 \pi$ can be noted.

\section{THE FRESNEL METASURFACE DESIGN}

The slit-shaped resonators designed in Sec. III can now be arranged in a non-periodic geometry to form the Fresnel metasurface, as shown in Fig. 8(a). Assuming the full $2 \pi$

Parametric Study of the periodic array of Fano resonators
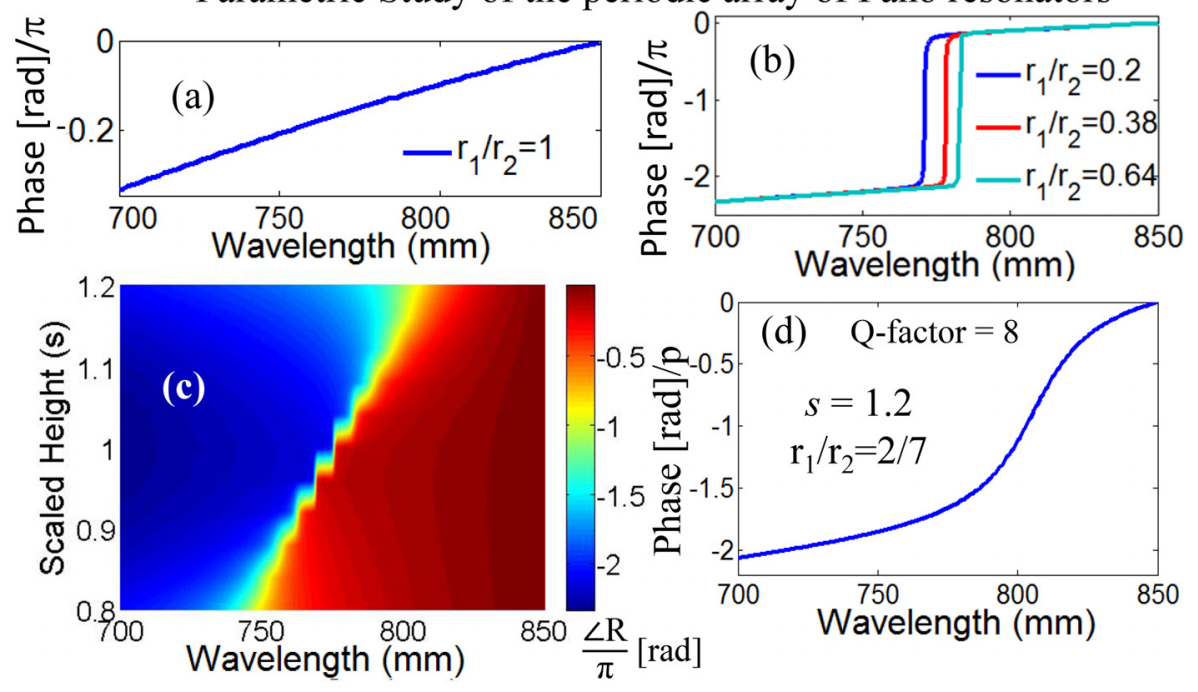

FIG. 6. (a) Reflection phase showing linear change because of the unit cell consists of identical resonators. (b) Reflection phase for various relative widths $\left(r_{1} / r_{2}\right)$ but equal heights $s=1$. (c) Phase profile of reflection coefficient (scaled height $\mathrm{s}$ vs wavelength) of the proposed periodic Fano resonator under normal incident conditions. The color scales represent the normalized reflected phase $\angle R / \pi$. Reflected phase vs wavelength for various scaled heights (d) phase profile for the case $s=1.2$, extracted from part (c). 


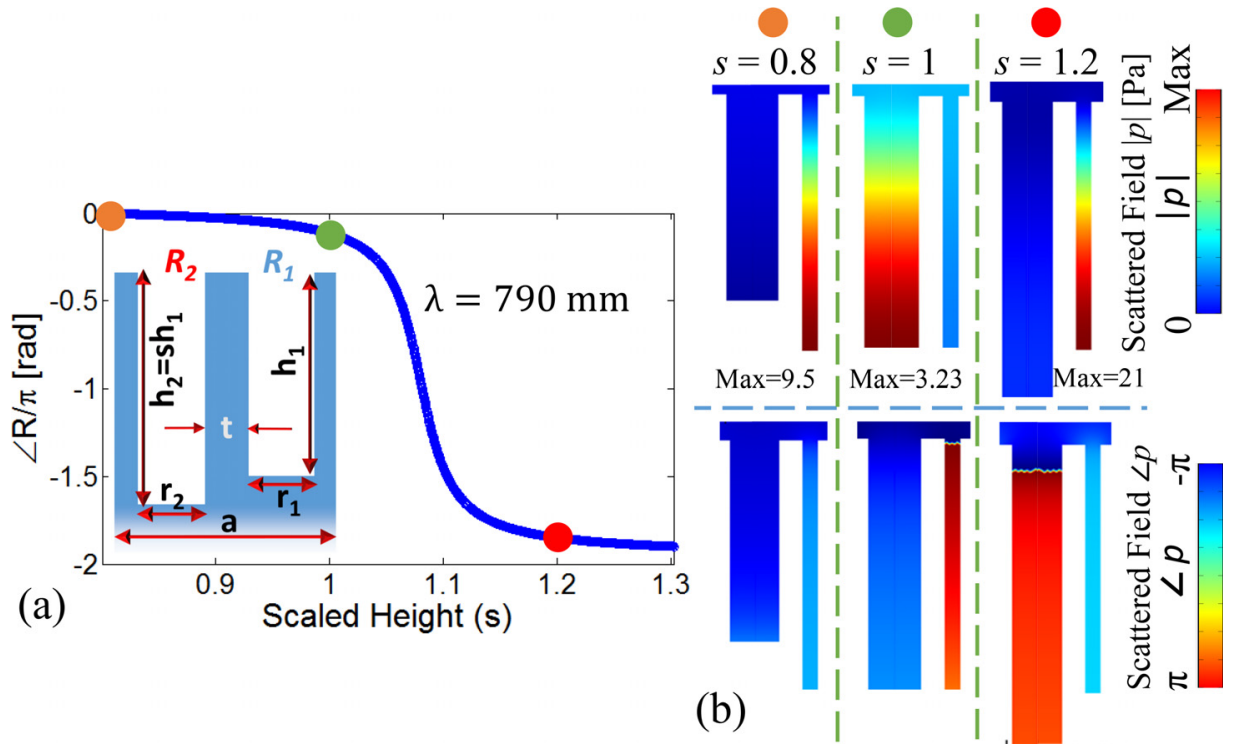

FIG. 7. (a) Reflected phase vs scaled height (s) at fixed incident wavelength of $790 \mathrm{~mm}$. (b) Scattered pressure field distribution at various scaled heights, i.e., $s=0.8,1$, and 1.2 , respectively. The field plots in the top and bottom correspond to $|p|$ and $\angle p$, respectively. The maximum of pressure field distribution is given for each case.

range of phase change across the metasurface and a focal point located at $F=\lambda$, the required phase profile is calculated from Eq. (5) and is depicted in Fig. 8(b). For a periodicity of $a=70 \mathrm{~mm}$, the required phase profile translates to 38 unit cells (meta-atoms) along the flat Fresnel metasurface. The corresponding scaling factor $s$, also shown in Fig. 8(b), is obtained after an extensive simulation study for each phase angle. The design is simplified by the fact that only the specification of the scaling factor $s$ is necessary to achieve the required unit cell phases; the rest of geometrical parameters remain fixed.

The designed metasurface is implemented in COMSOL by terminating both the left and the right side by the periodic boundary conditions, while in the traverse direction, the perfectly matched layer is applied to terminate the computational domain [see Fig. 8(a)]. The scattered pressure fields are computed for the normal incident plane waves. The simulation results are depicted in Fig. 8(c) where only the scattered field distribution is plotted and the incident waves are suppressed to avoid confusion. The clear formation of focus at the desired location $F=\lambda$ confirms the convergence of reflected sound waves through the constructive interference of the correctly phase-profiled metasurface cells. The inset in Fig. 8(c) shows the absolute value of the scattered field on the focal plane along the line perpendicular to the principle axis. As shown, the FWHM of the beam diameter reaches a maximum of $0.54 \lambda$ depicting the confinement of the acoustic waves into a narrow tight spot.

Next, we design the metasurface so that the focal point is shifted to the following three locations, i.e., $F=1.5 \lambda, 2 \lambda$, and $2.5 \lambda$. The number of unit cells that would guarantee a full $2 \pi$ phase change over the metasurface for the three cases are given by 44, 50, and 54, respectively. As shown in Figs. 9 (a) -9 (c), the scattered fields indeed interfere constructively to form the focal points at the designed locations. The respective values of the focal spot lengths (FWHM) for
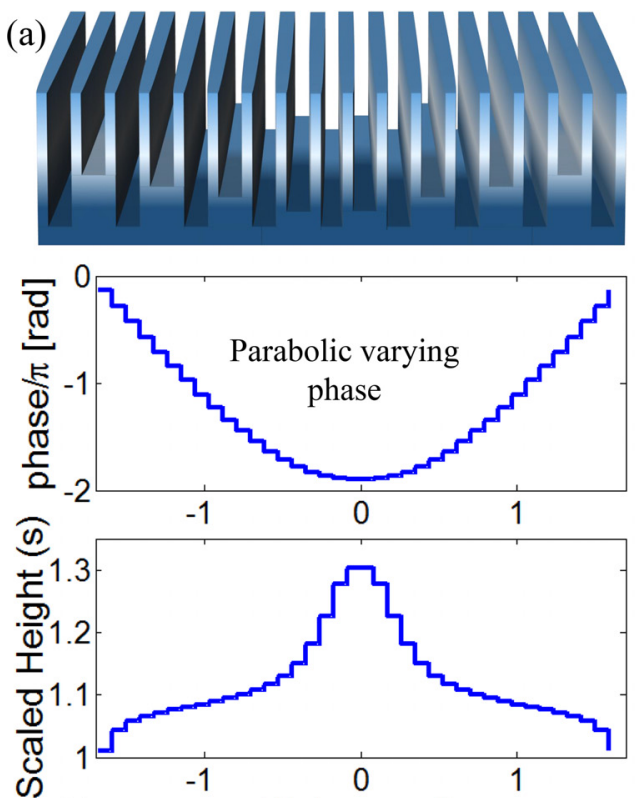

(b)
Distance $x / \lambda$

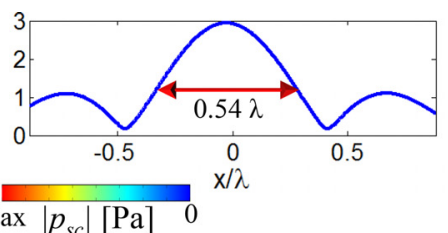

$$
\text { c) }
$$

(c)

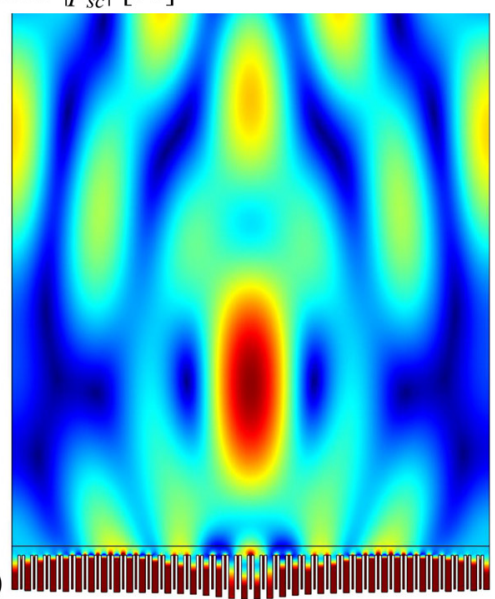

FIG. 8. (a) Schematic design of FanoFresnel metascreen lens based on detuned coupled resonances from resonators $R_{1}$ and $R_{2}$. (b) Required scattered field phase and corresponding scaled height (s) of resonator $R_{2}$ to achieve focal point (FL) at $\lambda$. Corresponding parabolic phase change of the reflected light is controlled from 0 to $-2 \pi$ by controlling resonator height $h_{2}=s h_{1}$. (c) Distribution of scattered fields $|p|$ of the Fano-Fresnel beam focusing metascreen. Inset graph plots the absolute value of scattered pressure in the traverse direction around focal length. The colorbar represents the amplitude of scattered field $\left|p_{s c}\right|$ between 0 and Max. Here, Max refers to maximum of the normalized scattered field around focal point provided in the inset. 

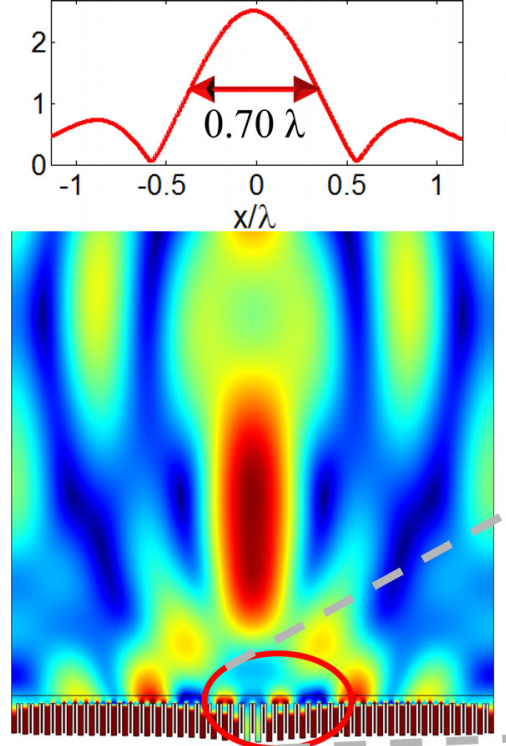

(a) Focal Length $=1.5 \lambda$
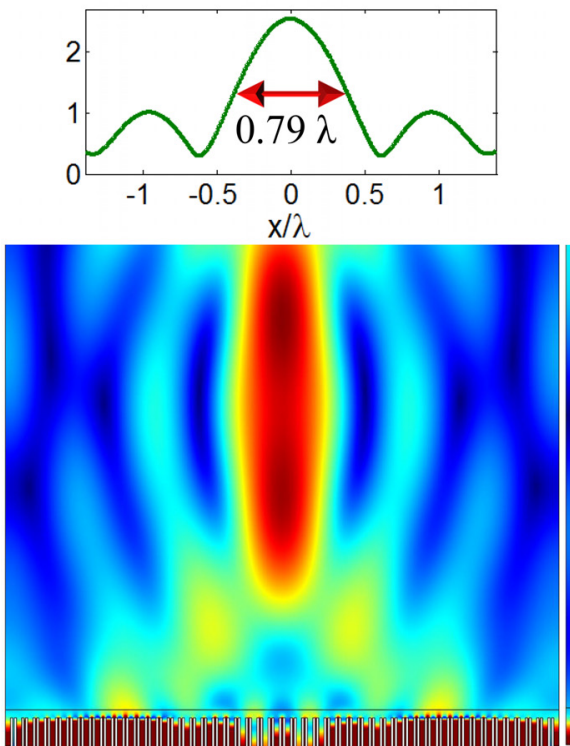

(b) Focal Length $=2 \lambda$
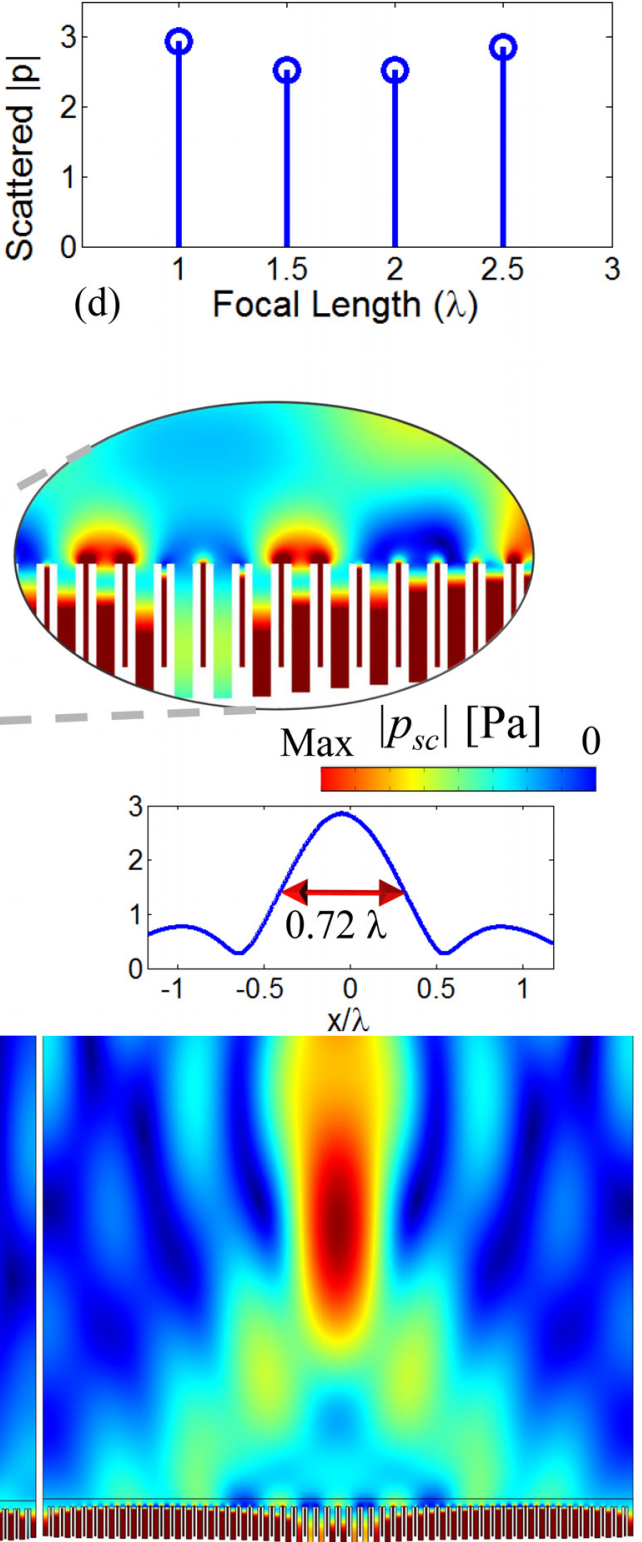

(c) Focal Length $=2.5 \lambda$
FIG. 9. (a) Scattered field distribution $|p|$ of the Fano-Fresnel beam focusing metascreen designed for various focal points (a) $\mathrm{FL}=1.5 \lambda$, (b) $\mathrm{FL}=2 \lambda$, and (c) $\mathrm{FL}=2.5 \lambda$ at an incident wavelength of $790 \mathrm{~mm}$. Inset in each subfigure represents the scattered field distribution in the lateral direction around Focal point for each designed metascreen. The lateral distance is scaled in reference to wavelength $\lambda$. (d) Plots for the absolute value of scattered field at focal point in various designed metascreen lenses. The colorbar represents the amplitude of scattered field $\left|p_{s c}\right|$ between 0 and Max. Here, Max refers to maximum of the normalized scattered field around each focal point in part (c). the three cases are given by $0.70 \lambda, 0.79 \lambda$, and $0.72 \lambda$. Lastly, the effective amplification can be compared by finding the relative magnitude of the scattered field at the focal point for various metasurface designs. The results depicted

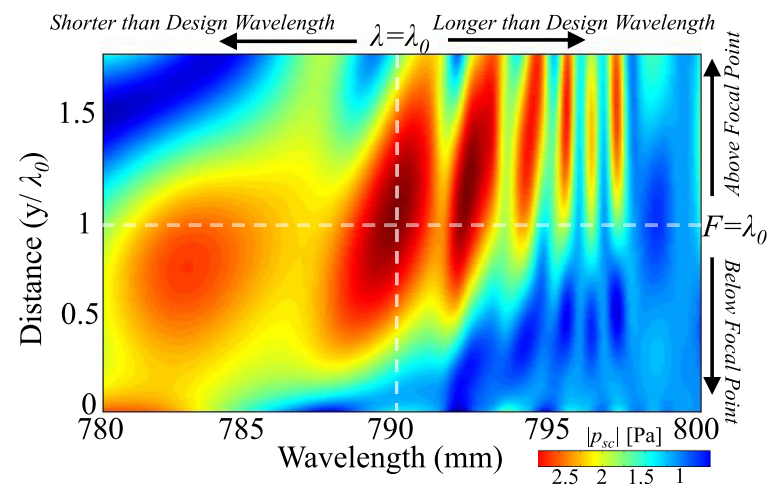

FIG. 10. The change in the amplitude of scattered pressure field along the distance $y / \lambda$ around principle axis of the lens at various frequencies. The design of lens is already described in Fig. 8. in Fig. 9(d) show that the metasurface offers a very high scattered field amplification that varies between a factor of 2.5 and 3 .

To characterize the effect of frequency variation, the scattering fields of the $790 \mathrm{~mm}$ metascreen (Fig. 8) are recalculated for a range of wavelengths between $780 \mathrm{~mm}$ and $800 \mathrm{~mm}$. The resulting 2D surface plot which summarizes the relation between the focal lengths and the operating wavelengths is depicted in Fig. 10. The results show familiar association of frequency-focus variation that has been previously observed in highly resonant metamaterial lenses. ${ }^{65-67}$ At $790 \mathrm{~mm}$, all the scattered acoustic waves follow the designed phase profile and hence are tightly focused around $F=\lambda_{0}$. Below the designed wavelength, the additional phase lag causes the scattered waves to bend closer to the reflector, leading to the formation of the focal point nearer to the Fresnel reflector. Note the broader spot appearing around $F=783 \mathrm{~mm}$ due to the aberration of the scattered fields. On the other hand, for the wavelengths above $790 \mathrm{~mm}$, there is 
an additional phase lead that incurs in the unit cells resulting in the appearance of foci away from the metascreen.

\section{CONCLUSION}

In conclusion, we propose a Fresnel acoustic metasurface that can reflect the planar wavefronts with perfect reflection characteristics. The metasurface is designed by replacing the curved parabolic shaped reflecting segments in the classical Fresnel reflector with double slit-shaped quarter wavelength cavity unit-cells. The dispersion of the metasurface is tailored according to the Huygens' principle in such a way that the planar incident wavefronts are converted to spherical wavefronts upon reflection from the metasurface. This is made possible primarily by the sharp-slope phase characteristics offered by Fano resonances. The unit cells can be designed according to the prescribed phase profile with slight detuning that is obtained by incremental modifications to adjacent unit cells, thus giving a flat and compact geometry to the metasurface. The efficient control over the reflected fields' phase is exploited to build readjustable flat Fresnel metascreen lenses. High field intensity at focal point together with tight focusing of scattered fields through such metascreen can be used for imaging applications.

${ }^{1}$ S. Datta, "Classical wave propagation in periodic and random media," Ph.D. thesis (Iowa State University, 1994).

${ }^{2}$ E. Yablonovitch, Phys. Rev. Lett. 58, 2059 (1987).

${ }^{3}$ S. John, Phys. Rev. Lett. 58, 2486 (1987).

${ }^{4}$ R. Zengerle, J. Mod. Opt. 34, 1589 (1987).

${ }^{5}$ T. F. Krauss, M. Richard, and S. Brand, Nature 383, 699 (1996).

${ }^{6}$ B. Gralak, S. Enoch, and G. Tayeb, JOSA A 17, 1012 (2000).

${ }^{7}$ M. Notomi, Opt. Quantum Electron. 34, 133 (2002).

${ }^{8}$ R. V. Craster and S. Guenneau, Acoustic Metamaterials: Negative Refraction, Imaging, Lensing and Cloaking (Springer Science and Business Media, 2012), Vol. 166.

${ }^{9}$ E. Lheurette, Metamaterials and Wave Control (John Wiley and Sons, 2013).

${ }^{10}$ Z. Liu, X. Zhang, Y. Mao, Y. Zhu, Z. Yang, C. T. Chan, and P. Sheng, Science 289, 1734 (2000)

${ }^{11}$ C. Poulton, A. Movchan, R. McPhedran, N. Nicorovici, and Y. Antipov, Proc. R. Soc. London, Ser. A 456, 2543-2559 (2000).

${ }^{12}$ S. Yang, J. H. Page, Z. Liu, M. L. Cowan, C. T. Chan, and P. Sheng, Phys. Rev. Lett. 93, 024301 (2004).

${ }^{13}$ L. Feng, X.-P. Liu, Y.-B. Chen, Z.-P. Huang, Y.-W. Mao, Y.-F. Chen, J. Zi, and Y.-Y. Zhu, Phys. Rev. B 72, 033108 (2005).

${ }^{14} \mathrm{X}$. Zhang and Z. Liu, Appl. Phys. Lett. 85, 341 (2004).

${ }^{15}$ X. Hu, Y. Shen, X. Liu, R. Fu, and J. Zi, Phys. Rev. E 69, 030201 (2004).

${ }^{16}$ M. Farhat, S. Guenneau, S. Enoch, G. Tayeb, A. Movchan, and N. Movchan, Phys. Rev. E 77, 046308 (2008).

${ }^{17}$ M. Farhat, S. Guenneau, S. Enoch, and A. Movchan, J. Comput. Appl. Math. 234, 2011 (2010).

${ }^{18}$ J. Page, A. Sukhovich, S. Yang, M. L. Cowan, F. Van Der Biest, A. Tourin, M. Fink, Z. Liu, C. T. Chan, and P. Sheng, Phys. Status Solidi B 241, 3454 (2004).

${ }^{19}$ Y. Pennec, J. O. Vasseur, B. Djafari-Rouhani, L. Dobrzyński, and P. A. Deymier, Surf. Sci. Rep. 65, 229 (2010).

${ }^{20}$ G. Dupont, M. Farhat, A. Diatta, S. Guenneau, and S. Enoch, Wave Motion 48, 483 (2011).

${ }^{21}$ M. D. Guild, M. R. Haberman, and A. Alù, Wave Motion 48, 468 (2011).

${ }^{22}$ P.-Y. Chen, M. Farhat, S. Guenneau, S. Enoch, and A. Alù, Appl. Phys. Lett. 99, 191913 (2011).

${ }^{23}$ A. N. Norris, Proc. R. Soc. London, Ser. A 464, 2411-2434 (2008).

${ }^{24}$ M. Farhat, S. Guenneau, and S. Enoch, Phys. Rev. B 85, 020301 (2012).
${ }^{25}$ T. Bückmann, M. Thiel, M. Kadic, R. Schittny, and M. Wegener, Nat. Commun. 5, 4130 (2014).

${ }^{26}$ J. Li and C. Chan, Phys. Rev. E 70, 055602 (2004).

${ }^{27}$ Y. Ding, Z. Liu, C. Qiu, and J. Shi, Phys. Rev. Lett. 99, 093904 (2007).

${ }^{28}$ S. Guenneau, A. Movchan, G. Pétursson, and S. A. Ramakrishna, New J. Phys. 9, 399 (2007).

${ }^{29}$ Z. Liang, T. Feng, F. L. S. Lok, K. B. Ng, C. H. Chan, J. Wang, S. Han, S. Lee, and J. Li, Sci. Rep. 3, 1614 (2013).

${ }^{30}$ J. B. Pendry, Phys. Rev. Lett. 85, 3966 (2000).

${ }^{31}$ S. A. Cummer and D. Schurig, New J. Phys. 9, 45 (2007).

${ }^{32}$ M. Fleischhauer, A. Imamoglu, and J. P. Marangos, Rev. Mod. Phys. 77, 633 (2005).

${ }^{33}$ N. Liu, L. Langguth, T. Weiss, J. Kästel, M. Fleischhauer, T. Pfau, and H. Giessen, Nat. Mater. 8, 758-762 (2009).

${ }^{34}$ M. Amin, R. Ramzan, and O. Siddiqui, Sci. Rep. 8, 2357 (2018).

${ }^{35}$ B. Luk'yanchuk, N. I. Zheludev, S. A. Maier, N. J. Halas, P. Nordlander, H. Giessen, and C. T. Chong, Nat. Mater. 9, 707-715 (2010).

${ }^{36}$ C. Wu, A. B. Khanikaev, R. Adato, N. Arju, A. A. Yanik, H. Altug, and G. Shvets, Nat. Mater. 11, 69-75 (2012).

${ }^{37}$ F. Liu, M. Ke, A. Zhang, W. Wen, J. Shi, Z. Liu, and P. Sheng, Phys. Rev. E 82, 026601 (2010).

${ }^{38}$ A. Santillán and S. I. Bozhevolnyi, Phys. Rev. B 84, 064304 (2011).

${ }^{39}$ M. Amin, A. Elayouch, M. Farhat, M. Addouche, A. Khelif, and H. Bağci, J. Appl. Phys. 118, 164901 (2015).

${ }^{40}$ W. L. Stutzman Boyd, Antenna Theory and Design (John Wiley and Sons, 2013).

${ }^{41}$ S. Wahlström, J. Audio Eng. Soc. 33, 418 (1985).

${ }^{42}$ C. Pfeiffer and A. Grbic, Phys. Rev. Lett. 110, 197401 (2013).

${ }^{43}$ M. Dubois, C. Shi, Y. Wang, and X. Zhang, Appl. Phys. Lett. 110, 151902 (2017).

${ }^{44}$ A. Díaz-Rubio and S. Tretyakov, Phys. Rev. B 96, 125409 (2017).

${ }^{45}$ N. Yu, P. Genevet, M. A. Kats, F. Aieta, J.-P. Tetienne, F. Capasso, and Z. Gaburro, Science 334, 333 (2011).

${ }^{46}$ A. Epstein and G. V. Eleftheriades, J. Opt. Soc. Am. B 33, A31 (2016).

${ }^{47}$ G. Ma, M. Yang, S. Xiao, Z. Yang, and P. Sheng, Nat. Mater. 13, 873 (2014).

${ }^{48}$ K. Song, M. Anzan-Uz-Zaman, J.-H. Kwak, J.-Y. Jung, J. Kim, and S. Hur, AIP Adv. 7, 035315 (2017).

${ }^{49}$ A. Davis and F. Kühnlenz, Opt. Photonik 2, 52 (2007).

${ }^{50}$ S. Guenneau and S. A. Ramakrishna, C. R. Phys. 10, 352 (2009).

${ }^{51}$ Y. Ding, E. C. Statharas, K. Yao, and M. Hong, Appl. Phys. Lett. 110, 241903 (2017).

${ }^{52}$ Y. Li, B. Liang, Z.-M. Gu, X.-Y. Zou, and J.-C. Cheng, Sci. Rep. 3, 2546 (2013).

${ }^{53}$ Y. Xie, W. Wang, H. Chen, A. Konneker, B.-I. Popa, and S. A. Cummer, Nat. Commun. 5, 5553 (2014).

${ }^{54}$ L. Zigoneanu, B.-I. Popa, and S. A. Cummer, Phys. Rev. B 84, 024305 (2011).

${ }^{55}$ M. Ruphuy, O. Siddiqui, and O. M. Ramahi, J. Opt. Soc. Am. A 32, 1700 (2015).

${ }^{56} \mathrm{P}$. Clemmow, The Plane Wave Spectrum Representation of Electromagnetic Fields (Pergamon Press Ltd, 1966).

${ }^{57}$ U. Fano, Phys. Rev. 124, 1866 (1961).

${ }^{58}$ C. Ott, A. Kaldun, P. Raith, K. Meyer, M. Laux, J. Evers, C. H. Keitel, C. H. Greene, and T. Pfeifer, Science 340, 716 (2013).

${ }^{59}$ M. Amin, M. Farhat, and H. Baci, Sci. Rep. 3, 2105 (2013).

${ }^{60}$ M. Amin, R. Ramzan, and O. Siddiqui, Appl. Phys. Lett. 110, 181904 (2017).

${ }^{61}$ A. E. Miroshnichenko, S. Flach, and Y. S. Kivshar, Rev. Mod. Phys. 82, 2257 (2010).

${ }^{62}$ M. Rahmani, B. Luk'yanchuk, and M. Hong, Laser Photonics Rev. 7, 329 (2013).

${ }^{63}$ M. Amin, M. Farhat, and H. Bağci, Opt. Express 22, 6966 (2014).

${ }^{64}$ M. O’Donnell, E. Jaynes, and J. Miller, J. Acoust. Soc. Am. 69, 696 (1981).

${ }^{65}$ A. Grbic and G. V. Eleftheriades, Phys. Rev. Lett. 92, 117403 (2004).

${ }^{66}$ O. Siddiqui and G. V. Eleftheriades, Appl. Phys. Lett. 85, 1292 (2004).

${ }^{67}$ G. V. Eleftheriades and O. F. Siddiqui, IEEE Trans. Microwave Theory Tech. 53, 396 (2005). 